\title{
FRATURAS DO RÁDIO DISTAL: AVALIAÇÃO DAS CLASSIFICAÇÕES
}

\author{
Osvaldo Mendes de Oliveira Filho, William Dias Belangero*, João Batista Mendes Teles \\ Trabalho realizado na Faculdade de Ciências Médicas da Universidade Estadual de \\ Campinas (Unicamp), Campinas, SP, e Centro de Ciências da Saúde da \\ Universidade Federal do Piauí, Teresina, Piauí, PI.
}

\begin{abstract}
RESUMO - OBjetivos. 0 objetivo deste trabalho foi determinar 0 grau de reprodutibilidade intra $e$ interobservador em relação às classificações de Frykman, AO e Universal para as fraturas distais do rádio.

Métodos. Neste estudo foram selecionadas 40 radiografias de fraturas distais do rádio que foram classificadas por ortopedistas de centros e nível de experiência diferentes, determinando-se 0 grau de reprodutibilidade intra e interobservador através do método estatístico Kappa.
\end{abstract}

Resultados. A concordância intra-observador média observada foi moderada para as classificações de Frykman e Universal e leve para o sistema AO. A reprodutibilidade média interobservador foi leve nas duas leituras para as classificações de Frykman e Universal e desprezível na segunda leitura da classificação $\mathbf{A O}$.

Conclusäo. Todas as classificações utilizadas apresentaram reprodutibilidade interobservador questionável, comprometendo 0 uso dos três sistemas avaliados

UnItermos: Classificação. Rádio distal. Fraturas. Reprodutibilidade.

\section{INTRODUÇÃO}

As fraturas da extremidade distal do rádio são definidas como aquelas que ocorrem a até $3 \mathrm{~cm}$ da articulação rádiocárpica!. São de grande importância e interesse, pois correspondem a um sexto de todas as fraturas atendidas nas salas de emergências. Nos Estados Unidos e no Reino Unido uma em cada grupo de 500 pessoas sofre essa fratura por ano, causando um alto custo socioeconômico. A faixa etária mais acometida é entre 60 e 69 anos, principalmente em mulheres, mas nota-se uma elevação da prevalência entre jovens devido aos acidentes de trânsito e traumas esportivos ${ }^{2}$.

Para se determinar o perfil radiográfico das fraturas do rádio distal são necessárias radiografias de boa qualidade nas incidências ântero-posterior e lateral. Três medidas relacionadas ao eixo longitudinal do rádio podem ser avaliadas e servem de parâmetros para a redução. A inclinação volar da superfície articular do rádio varia de $11^{\circ}$ a $12^{\circ}$, sendo observada na incidência em perfil. A inclinação radial tem, em média, de 22- a 23-, e, juntamente

* Correspondência:

Rua Emílio Ribas, 800 - Apt. 1

Cep: 13025-141 - Campinas - SP

Fone: (19) 3788-7750/3254-0220

E-mail: belanger@sigmanet.com.br com altura radial, que mede de $1 \mathrm{I}$ a $12 \mathrm{~mm}$, são avaliadas na incidência ântero-posterior ${ }^{3}$. São consideradas instáveis aquelas fraturas que apresentam grande desvio e cominuição dorsal, angulação dorsal maior que $20^{\circ}$ - do fragmento distal, cominuição articular importante e encurtamento do fragmento distal maior que $10 \mathrm{~mm}$. Fraturas com essas características tendem a perder a redução ${ }^{4}$.

Certos padrões de fraturas como fraturasluxações rádio-cárpicas e fraturas do processo estilóide radial muito desviadas têm, obrigatoriamente, lesões concomitantes de ligamentos intrínsecos ou extrínsecos do carpo, que devem ser consideradas quando classificamos as fraturas do rádio distal, uma vez que influenciarão no resultado ${ }^{5}$.

Existem inúmeras classificações para fraturas da extremidade distal do rádio. Segundo Müller et al. (1987), qualquer sistema de classificação realmente útil deve considerar o tipo e a gravidade da fratura, servir como base para o tratamento e de guia para a avaliação do resultado 6.

Frykman (1967) estabeleceu uma classificação que levava em conta o envolvimento ou não da articulação rádio-cárpica e rádio-ulnar e a presença ou ausência de fratura do processo estilóide ulnar. Apesar de ser mundialmente conhecida e muito citada na literatura, falha porque não considera o desvio inicial da fratura, nem o encurtamento do rádio.
A classificação de Frykman tem uma importância fundamental por ter chamado a atenção à ulna distal e à articulação rádio-ulnar distal, muitas vezes relegada a um segundo plano durante a redução ${ }^{7}$ (Figura I).

A classificcação AO foi criada em 1986 e revisada em 1990. Ela considera a gravidade da lesão óssea e serve como base para o tratamento e avaliação dos resultados. Existem três tipos básicos: extra-articular, articular parcial e articular completa. Os três grupos são organizados em ordem crescente de gravidade com relação à complexidade morfológica, dificuldade de tratamento e prognóstico. É, sem dúvida, a classificação mais completa, mas sua reprodutibilidade intra e interobservador tem sido um problema quando o grupo e subgrupo estão sendo avaliados ${ }^{8,9}$. Às vezes, não é possivel determinar todas as linhas de fraturas através de radiografias simples, sendo necessárias incidências radiográficas especiais ou tomografia. O grupo A (extra-articular) não envolve a articulação rádio-cárpica, o grupo B (fraturas articulares parciais) envolve a articulação rádio-cárpica, mas uma porção da superfície articular permanece em continuidade com a diáfise e o grupo C (articular completa) apresenta separação completa da articulação com a diáfise. Estes três principais tipos são subdivididos em três grupos, e cada grupo, em três subgrupos. Dessa forma, existem 27 diferentes padrões de fraturas que dependem da 


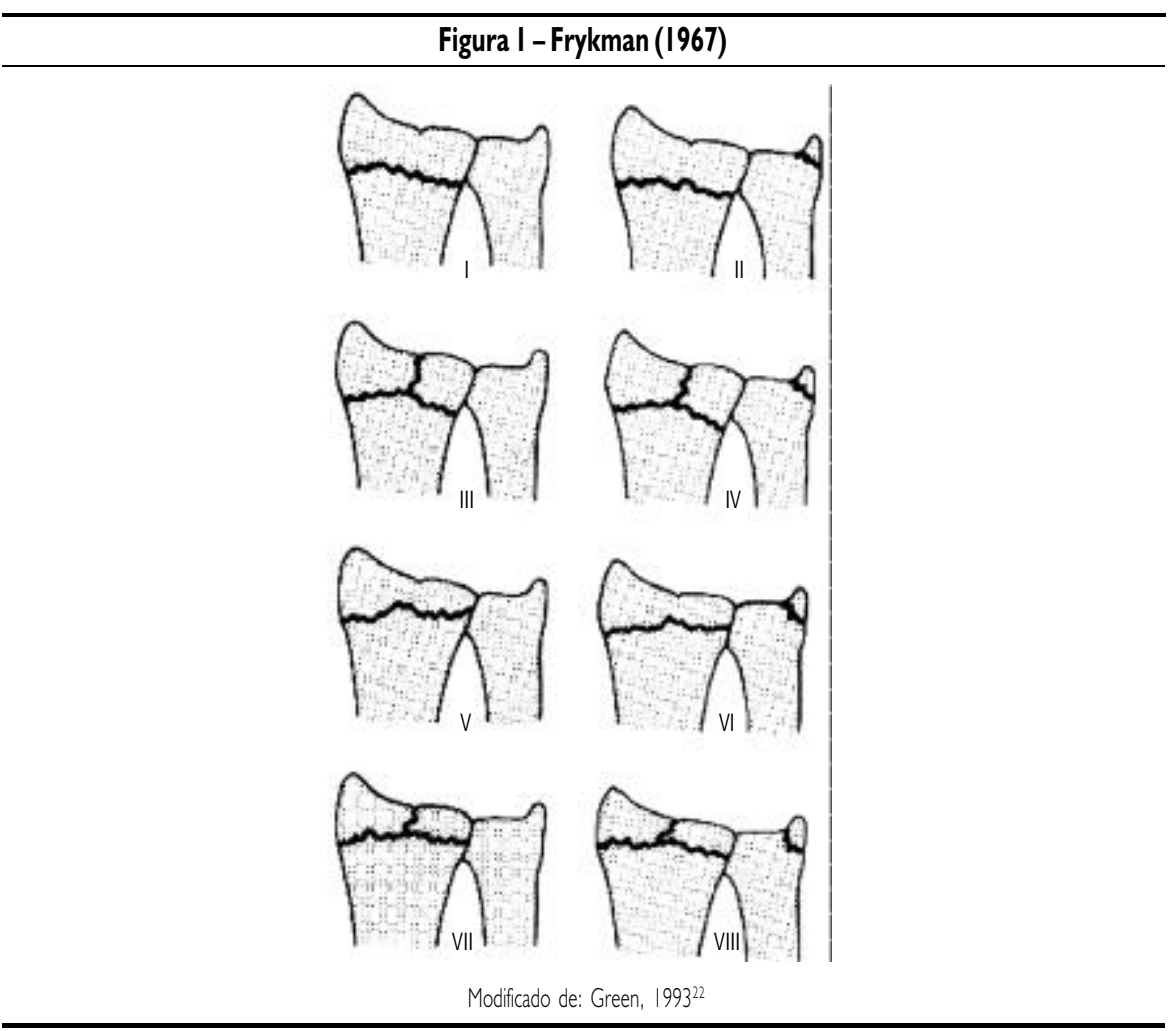

Figura 2-AO(1987)
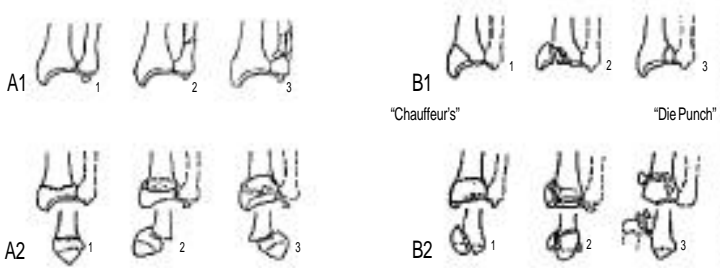

Chauffeu's

"DiePunch"
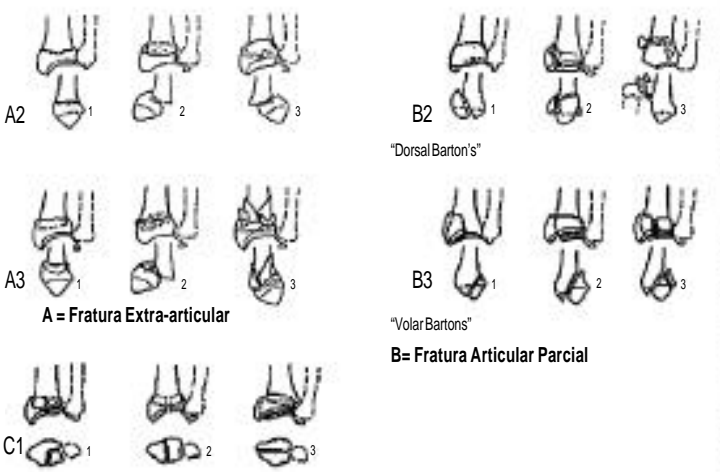

B= Fratura Articular Parcial
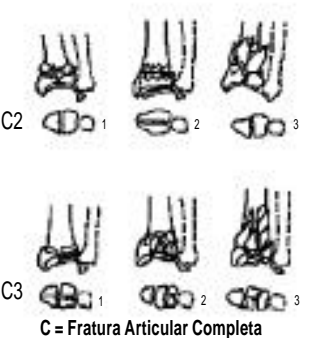

Modificado de: Kreder et al, $1996^{\circ}$ estabilidade, impacto, cominuição, redutibilidade e localização dos fragmentos 2,10 . Esta classificação é a adotada pela Associação de Trauma Ortopédico dos Estados Unidos e uma das mais usadas em todo o mundo" (Figura 2).

A classificação Universal ou de Rayhack (1990) foi criada em um simpósio sobre fraturas distais do rádio em 1990 e modificada por Cooney em 1993. Diferenciava as fraturas intra e extra-articulares, desviadas ou não, e a redutibilidade e estabilidade das fraturas. As extra-articulares são chamadas tipo I (estável, sem desvio) e tipo II (instável, com desvio). E as intraarticulares são também, da mesma forma, subdivididas em tipo III (estável, sem desvio) e tipo IV que apresenta os subtipos: $A$, estável e redutível; $B$, redutível e instável; C, irredutível e D, complexa ${ }^{12}$ (Figura 3).

Ainda não temos uma classificação ideal para as fraturas da extremidade distal do rádio, que nos permita uma visão sistêmica do punho, de sua complexa anatomia e biomecânica, de sua relaçãa com o carpo e partes moles. A classificação ideal deve permitir escolher um tratamento adequado, determinar o prognóstico, comparar modalidades de tratamento, deve ser de fácil memorização, ser internacionalmente aceita e ter uma reprodutibilidade satisfatória ${ }^{9}, 10,11$.

Como afirmou Bernstein ${ }^{13}$, é impossível satisfazer todas essas informações em um único sistema, mas o estudo profundo das classificações é de grande importância para se obter um melhor tratamento dos pacientes.

\section{Objetivo}

O objetivo deste trabalho é determinar o grau de reprodutibilidade intra-observador e interobservador em relação às classificações de Frykman, $\mathrm{AO}$ e Universal para as fraturas distais do rádio, analisadas em radiografias nas incidências ântero-posterior e lateral.

\section{Métodos}

Foram analisadas 40 radiografias de pacientes com fraturas distais do rádio. Os 40 aspectos radiográficos foram escolhidos em comum acordo com o orientador, procurando cobrir todo o espectro das classificações de Frykman, AO e Universal das fraturas distais do rádio com pelo menos um exemplar. 


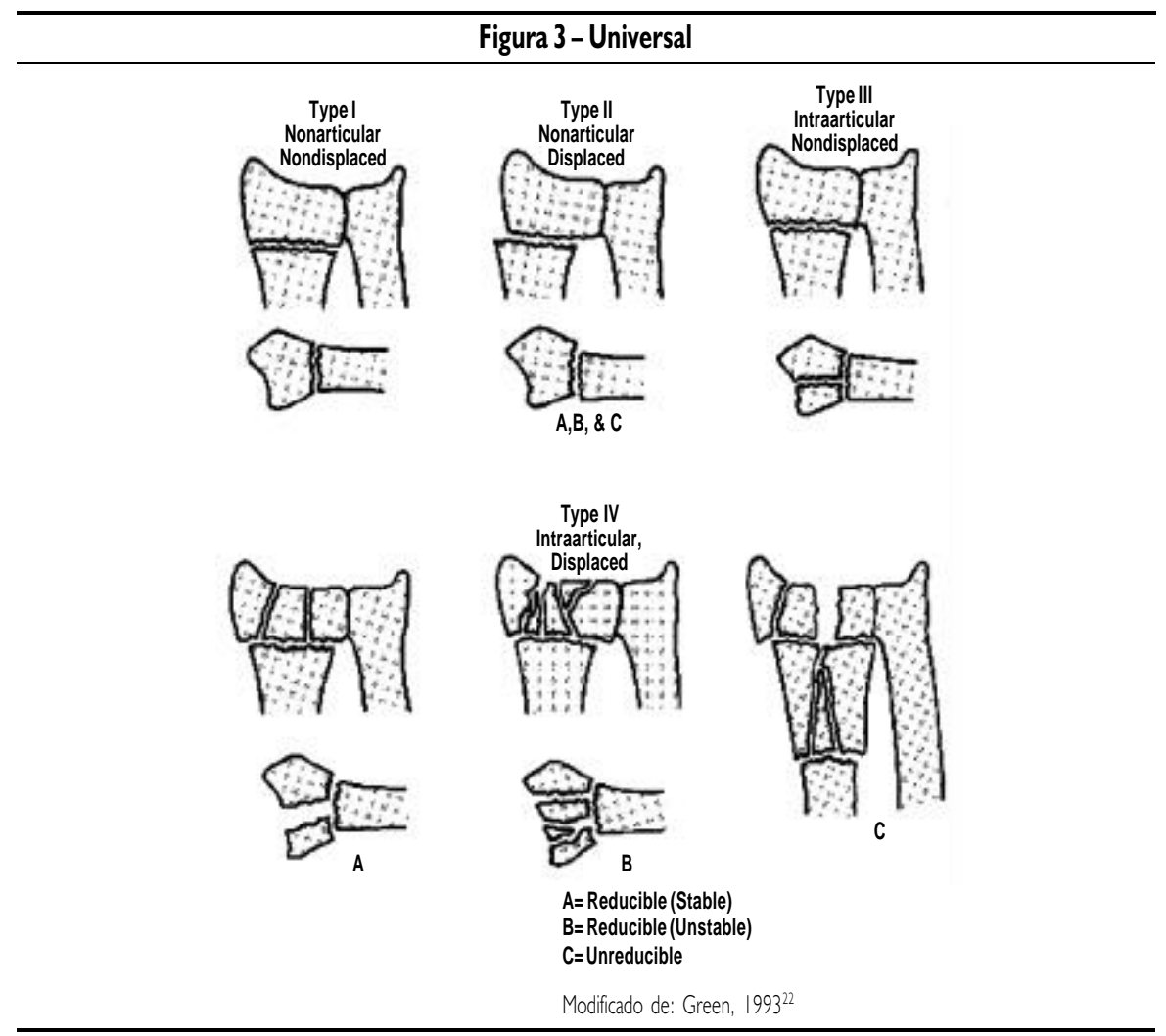

As radiografias foram obtidas antes da redução, nas incidências ântero-posterior e lateral, e todas foram fotografadas em câmera digital (Nikon, colpix 995) e copiadas em mídia digital (CD), sendo exibidas em sessões separadas a cada observador.

Inicialmente foi feita uma revisão de 30 minutos das classificações de Frykman, $\mathrm{AO}$ e Universal com cada participante, enfatizando os pontos importantes de cada classificação. Além disso, cada participante recebeu uma ilustração com todos os tipos das classificações utilizadas.

As radiografias foram examinadas por grupos de ortopedistas com diferentes graus de experiência, sendo a maioria deles familiarizadas com as classificações empregadas no estudo.

Os observadores foram constituídos por dois ortopedistas cirurgiões de mão (CMA e CMB); dois ortopedistas gerais, professores universitários ( OPA e OPB ); dois ortopedistas gerais sem ligação com instituições universitárias (OGA e OGB); dois residentes de ortopedia do terceiro ano (R3A e R3B) e dois residentes do segundo ano (R2A e R2B).

As radiografias avaliadas foram exatamente as mesmas para todos os participantes e foram classificadas em duas épocas diferentes: momento I e momento II. A ordem das radiografias na segunda sessão de classificação foi mudada e foi criada uma ficha de dados para que os participantes registrassem suas respostas nos dois momentos avaliados. Todos os integrantes classificaram as 40 radiografias segundo as classificações de Frykman, AO e Universal.

\section{Método estatístico}

método estatístico Kappa foi utilizado para analisar a reprodutibilidade intra e interobservadores entre os pares de observadores. O coeficiente Kappa (calculado pela fórmula: $\left.K=p_{0}-p_{e} / I-p_{e}\right)$ avalia a concordância entre os observadores através de análise pareada, comparando a proporção de concordância entre os observadores $\left(p_{0}\right)$ com a percentagem de concordância devido ao acaso $\left(p_{e}\right)^{14}$. A concordância real subtraída da aleatória, casual é I- p. A interpretação dos valores Kappa foi feito de acordo com o proposto por Landis e Koch'5, segundo os quais, valores Kappa menores que zero representam uma má reprodutibilidade, de zero a 0,20 desprezível, 0,21 a 0,40 reprodutibilidade leve, 0,41 a 0,60 reprodutibilidade moderada, 0,61 a 0,80 reprodutibilidade grande e acima de 0,80 considera-se uma concordância quase perfeita.

A reprodutibilidade interobservador refere-se ao nível de concordância entre diferentes observadores e a reprodutibilidade intra-observador representa o nível de concordância de um mesmo observador em ocasiões diferentes. Os valores obtidos da estatística Kappa foram testados em nível de significância de 5\% e os dados foram elaborados e analisados estatisticamente através do Programa StatXact, versão 3. $\left.\right|^{16}$.

\section{Resultados}

Neste estudo foram avaliadas três das classificações mais utilizadas para as fraturas da extremidade distal do rádio: Frykman, $\mathrm{AO}$ e Universal. Foram testadas a reprodutibilidade intra e interobservador de todas elas e também se o grau de experiência dos observadores melhora esses índices de concordância.

A classificação de Frykman, descrita em 1967, é a mais utilizada e, portanto, a que mais foi testada em toda a literatura mundial.

A concordância média intra-observador da classificação de Frykman obtida no nosso estudo foi moderada, com o valor Kappa médio de 0,5484 (Tabela I).

Houve uma tendência de melhora na concordância intra-observador à medida que se aumentava a experiência dos observadores.

A concordância média interobservador, todavia, mostrou-se leve, com o índice Kappa médio de 0,3742 no primeiro momento (Exemplo I). No momento dois, a concordância obtida também foi leve, com Kappa médio de 0,3424. Não houve melhora da reprodutibilidade interobservador nos momentos I e II, relacionada com a experiência dos entrevistados (Tabela 2). Quando avaliamos a concordância interobservador nas duas leituras, verificamos que não houve melhora da concordância se os residentes estavam ou não presentes (Tabela 3).

A classificação AO, descrita em 1987, é também uma das mais adotadas e testada em sua reprodutibilidade. 


\begin{tabular}{lcccccc}
\hline \multicolumn{3}{c}{ Tabela I } & - Concordância média intra-observador nas classificações Frykman, AO e Universal \\
\hline Classificação & $\begin{array}{l}\text { Kappa } \\
\text { médio }\end{array}$ & Concordância & $\begin{array}{c}\text { Kappa } \\
\text { médio } \\
\text { sem }\end{array}$ & Concordância & $\begin{array}{c}\text { Kappa } \\
\text { médio } \\
\text { apenas }\end{array}$ & Concordância \\
Frykman & 0,5484 & Moderada & 0,6103 & Grande & 0,4555 & Moderada \\
AO & 0,3815 & Leve & 0,4418 & Moderada & 0,2912 & Leve \\
Universal & 0,5423 & Moderada & 0,6171 & Grande & 0,4303 & Moderada \\
\hline
\end{tabular}

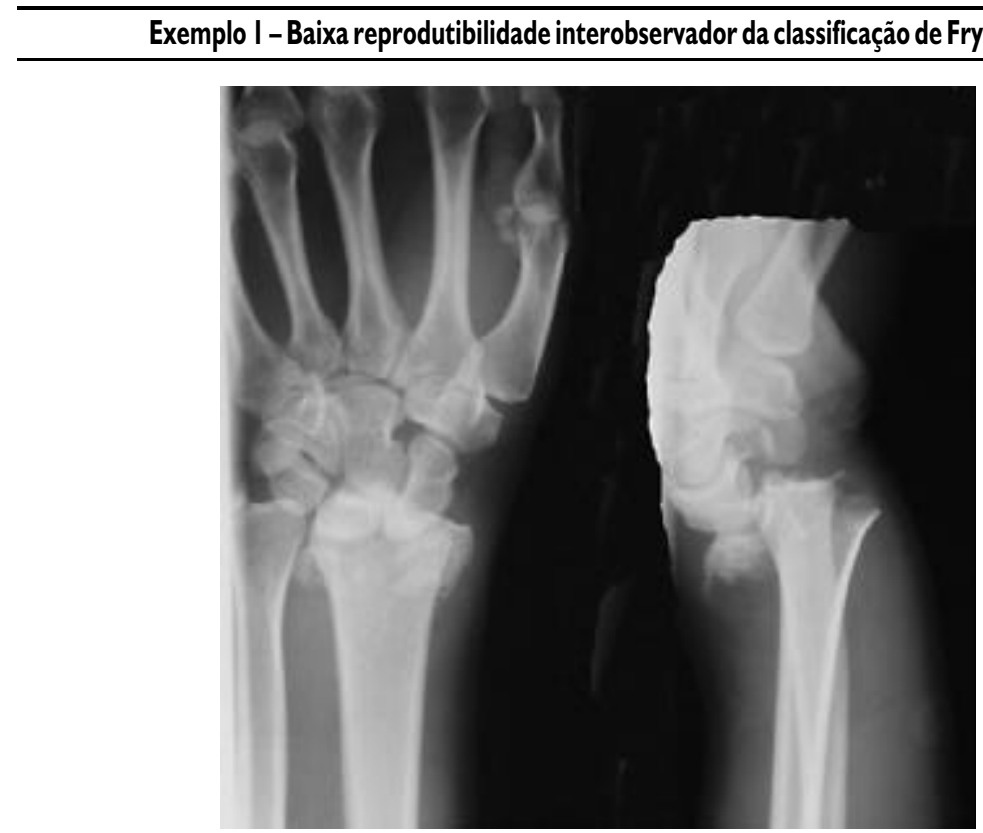

R2A: I; R2B: VIII; R3B: II; OGA: Vl; OGB: V; OPA: Vl; OPB: VI; CMA: V; CMB: VIII.

Vários estudos já confirmaram a reprodutibilidade da classificação $A O$, quando se considera os seus três "tipos" básicos, porém poucos provaram a concordância ou reprodutibilidade quando todos os "grupos" e "subgrupos" são considerados por múltiplos observadores. Dessa forma, nosso trabalho tem como um dos objetivos avaliar o sistema AO e também as outras classificações de forma completa 9 , 10 .

A concordância intra-observador da classificação AO foi apenas leve, obtendo-se o índice Kappa médio de 0,3815. Foi observada melhora da reprodutibilidade intra-observador quando os residentes foram excluídos, passando a uma concordância média moderada (Tabela I).

A reprodutibilidade interobservador do sistema $\mathrm{AO}$ foi a que apresentou maior número de concordâncias desprezíveis $(p>0,05)$. No primeiro momento, entretanto, alcançou-se uma concordância leve interobservador com valor Kappa médio de 0,2153. No segundo momento, a concordância média foi desprezível, com o valor Kappa médio de 0, 1788 (Tabela 2).

Constatamos, como já relatado em outros estudos, que nas duas sessões para avaliação da concordância interobservador houve uma grande variabilidade nas concordâncias, não havendo, nos dois momentos, qualquer indício de melhora da concordância com o aumento da experiência dos observadores avaliados 9.11 (Exemplo 2).

A classificação Universal, descrita em 1990, caracteriza-se pela simplicidade e funciona como guia de conduta para o tratamento. Chama-se Universal pelo fato de poder adaptar-se a outras classificações quando ela não suprir todas as situações das fraturas. Esta classificação não possui, até o momento, outros estudos da sua acurácia e reprodutibilidade.

Nossos observadores, mesmo conhecendo os critérios definidos de instabilidade das fraturas da extremidade distal do rádio, referiram dificuldade para afirmar se algumas fraturas eram ou não redutíveis. Isto se deveu, em parte, porque eles analisaram apenas as radiografias iniciais, portanto, antes da redução.

A concordância intra-observador alcançada na classificação Universal foi moderada, obtendo um índice Kappa médio de 0,5423 (Tabela I). Estes resultados foram equivalentes ao da classificação de Frykman.

Houve uma melhora na concordância à medida que passamos aos ortopedistas mais experientes. Ao excluirmos os residentes, a concordância intra-observador aumentou o seu valor Kappa médio $(K=0,6|7|)$, passando a ter uma grande concordância (Tabela 2).

A reprodutibilidade interobservador da classificação Universal foi leve, com um valor Kappa médio de 0,3308 no primeiro momento e 0,3275 no segundo momento, portanto, melhor que a classificação $\mathrm{AO}$ e semelhante à classificação de Frykman (Tabela 2).

Nas duas leituras da concordância interobservador da classificação Universal, à exemplo da classificação $A O$, consta tou-se uma grande variabilidade dos resultados, também não havendo influência da experiência dos observadores neste tipo de reprodutibilidade (Exemplo 3). Comparando-se a concordância interobservador nos dois momentos, constatamos que o valor Kappa médio permanece leve nas duas ocasiões, independentemente da presença ou ausência dos residentes (Tabelas 3 e 4).

No segundo momento da avaliação da concordância interobservador não fizemos mais revisão das classificações, apenas os observadores consultavam as ilustrações com as classificações. Talvez, por isso, houve piora nos índices Kappa médio na segunda leitura de todas os sistemas avaliados. 


\begin{tabular}{|c|c|c|c|c|}
\hline \multirow[t]{2}{*}{ Classificação } & \multicolumn{2}{|c|}{ Momentol } & \multicolumn{2}{|c|}{ Momentoll } \\
\hline & Kappa médio & Concordância & Kappa médio & Concordância \\
\hline Frykman & 0,3742 & Leve & 0,3424 & Leve \\
\hline AO & 0,2153 & Leve & 0,1788 & Desprezível \\
\hline Universal & 0,3308 & Leve & 0,3275 & Leve \\
\hline
\end{tabular}

\section{Exemplo 2 - Baixa reprodutibilidade interobservador da classificação $\mathrm{AO}$}

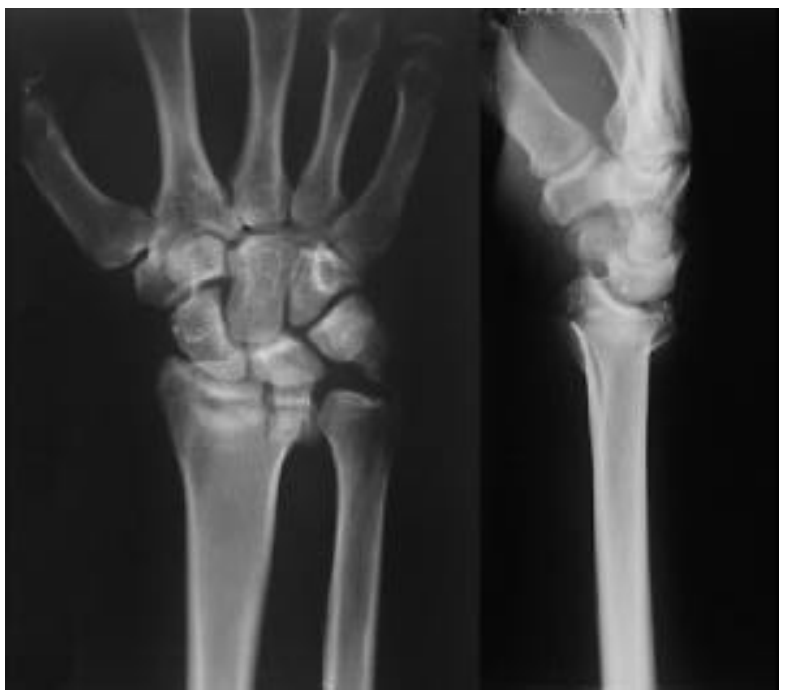

R2A: CI.2; R2B: A2.3; R3A: CI.I; R3B: A2.3; OGA: A3.2; OGB: A2.2; OPA: A3.I; OPB: CI.I; CMA: AI.I; CMB: A3.2.

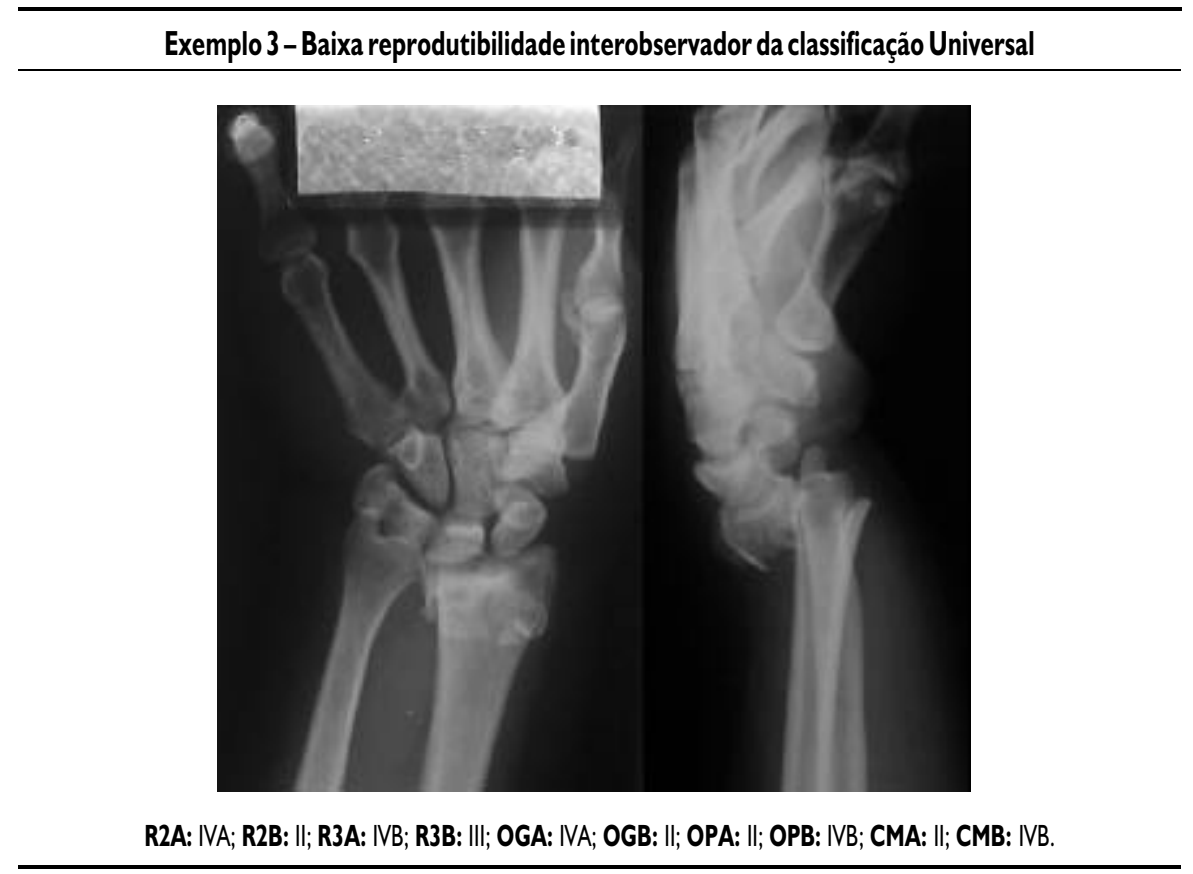

\section{Discussão}

Qualquer classificação deve ter uma boa reprodutibilidade intra e interobservador para que possa ser largamente aceita e permitir que diferentes séries sejam comparadas 9,17 .

Andersen et al.(1996) $)^{18}$ estudaram quatro classificações para as fraturas distais do rádio: Frykman, Melone, Mayo e AO. Verificaram que nenhuma delas mostrou grande concordância interobservador $(K=0,61$ a 0,80$)$. $\mathrm{Na}$ classificação de Frykman, a concordância intraobservador variou de 0,40 a 0,60 e a interobservador teve o índice Kappa médio de 0,36. Com relação ao sistema $A O$ completo, a concordância média intra-observador variou de 0,22 a 0,37 e, quando reduzida para três categorias, obteve-se um nível de concordância médio de 0,58 a 0,70. Contudo, reduzindo para três categorias, o sistema $\mathrm{AO}$ passa a ter valor questionável frente às outras classificações. A concordância média interobservador da classificação AO completa foi 0,25 e simplificada, 0,63.

Avaliando a reprodutibilidade do sistema AO em 30 radiografias de fraturas distais do rádio classificadas por 36 observadores com diferentes níveis de experiência, Kreder et al. $(1996)^{9}$ mostraram que a concordância interobservadores foi melhor para o "tipo" $\mathrm{AO}$ $(K=0,68)$ e decresceu progressivamente ao passar para os "grupos" $(K=0,48)$ e "subgrupos" $(K=0,33) A O$. Não houve diferença em relação ao grau de experiência dos observadores ao classificarem os "grupos" e "subgrupos". O índice Kappa médio variou de 0,25 a 0,42 na concordância intra-observador com o sistema $\mathrm{AO}$ completo e de 0,40 a 0,86 na classificação simplificada.

Illarramends et al. (1998) ${ }^{19}$ utilizaram 200 radiografias de fraturas distais do rádio, que foram classificadas por seis observadores com níveis diferentes de experiência. Para a classificação de Frykman, obtiveram reprodutibilidade interobservador moderada (Kappa médio de 0,43) e boa reprodutibilidade intra-observador (Kappa médio de 0,6I). Para a classificação $A O$, encontraram uma leve reprodutibilidade interobservador (Kappa $=0,37)$ e moderada intra-observador ( Kappa $=0,57$ ).

Para a obtenção de tais resultados, entretanto, os autores simplificaram as classificações de Frykman e AO, melhorando a 
Tabela 3 - Concordância média interobservador nas classificações de Frykman, AO e Universal, no momento I, com e sem a presença dos residentes

\begin{tabular}{|c|c|c|c|c|}
\hline \multirow[t]{2}{*}{ Classificação } & \multicolumn{2}{|c|}{ Momentol } & \multicolumn{2}{|c|}{ Momentol-Sem residentes } \\
\hline & Kappa médio & Concordância & Kappa médio & Concordância \\
\hline Frykman & 0,3742 & Leve & 0,3795 & Leve \\
\hline AO & 0,2153 & Leve & 0,2084 & Leve \\
\hline Universal & 0,3308 & Leve & 0,3696 & Leve \\
\hline
\end{tabular}

Tabela 4 - Concordância média interobservador nas classificações de Frykman, AO e Universal, no momento II, com e sem a presença dos residentes

\begin{tabular}{|c|c|c|c|c|}
\hline \multirow{2}{*}{ Classificação } & \multicolumn{2}{|c|}{ Momentoll } & \multicolumn{2}{|c|}{ Momentoll-Sem residentes } \\
\hline & Kappa médio & Concordância & Kappa médio & Concordância \\
\hline $\begin{array}{l}\text { Frykman } \\
\text { AO } \\
\text { Universal }\end{array}$ & $\begin{array}{l}0,3424 \\
0,1788 \\
0,3275\end{array}$ & $\begin{array}{c}\text { Leve } \\
\text { Desprezível } \\
\text { Leve }\end{array}$ & $\begin{array}{l}0,3602 \\
0,1726 \\
, 03576\end{array}$ & $\begin{array}{c}\text { Leve } \\
\text { Desprezível } \\
\text { Leve }\end{array}$ \\
\hline
\end{tabular}

Tabela 5 - Comparação da reprodutibilidade da classificação $\mathrm{AO}$ entre diferentes autores

\begin{tabular}{|c|c|c|c|c|}
\hline \multirow[t]{2}{*}{ Autor } & \multicolumn{2}{|c|}{$\begin{array}{c}\text { AO } \\
\text { Intra-observador } \\
\end{array}$} & \multicolumn{2}{|c|}{$\begin{array}{c}\text { AO } \\
\text { Interobservador }\end{array}$} \\
\hline & Completa & Simplificada & Completa & Simplificada \\
\hline Andersen et al., 1996 & $\begin{array}{c}0,22 \\
a \\
0,37\end{array}$ & $\begin{array}{c}0,58 \\
a \\
0,70\end{array}$ & 0,25 & 0,63 \\
\hline Kreder et al., 1998 & $\begin{array}{c}0,25 \\
a \\
0,42\end{array}$ & $\begin{array}{c}0,40 \\
a \\
0,86\end{array}$ & 0,33 & 0,68 \\
\hline Mendes \& Belangero, 2003 & 0,38 & 0,61 & 0,21 & 0,50 \\
\hline
\end{tabular}

\begin{tabular}{lcc}
\hline \multicolumn{2}{c}{ Tabela 6-Comparação da reprodutibilidade da classificação de Frykman entre diferentes autores } \\
\hline Autor & $\begin{array}{c}\text { Frykman } \\
\text { Intra-observador }\end{array}$ & $\begin{array}{c}\text { Frykman } \\
\text { Interobservador }\end{array}$ \\
\cline { 2 - 3 } Andersen et al., 1996 & $0,40 \mathrm{a} 0,60$ & 0,36 \\
Illarramends et al., 1998 & 0,61 & 0,43 \\
Mendes \& Belangero, 2003 & 0,54 & 0,37 \\
\hline
\end{tabular}

reprodutibilidade de ambas, o que talvez não ocorreria se estivessem completas. Houve maior reprodutibilidade intra-observador que interobservador e a concordância não melhorou com o aumento da experiência do observador.

Os autores concluíram que as classificações de Frykman e AO não são recomendadas para aplicação clínica devido à questionável reprodutibilidade de ambas as classificações.
Flinkkilä et al. $(1998)^{20}$ encontraram reprodutibilidade pobre quando usaram o sistema AO completo para classificar 30 radiografias com fraturas distais do rádio. Contudo, houve melhora quando reduziram a classificação AO para apenas dois tipos (extra e intra-articulares). Ao acrescentarem tomografias computadorizadas às radiografias planas também não se observou melhora na reprodutibilidade.
O uso da tomografia pode ser feito quando houver limitações da radiografia simples para os casos mais graves, entretanto, o custo para isso, no nosso meio, é proibitivo.

Oskam et al. (200 I)" estudaram a concordância da classificação $A O$ para as fraturas distais do rádio, utilizando apenas as três categorias básicas (A, B e C). Dois observadores experientes classificaram as 124 fraturas. Cada observador classificou isoladamente todas as radiografias obtendo um valor Kappa médio de 0,65 (boa concordância). Em seguida, numa reunião de consenso, classificaram as radiografias conjuntamente e obtiveram um valor Kappa de 0,86 (excelente concordância). $\mathrm{Na}$ reunião de consenso os pontos de discordância foram identificados e discutidos, e após essa revisão em conjunto o nível de concordância aumentou $12 \%$, passando de $80 \%$ para 92\%. Concluíram que o ponto de partida para melhorar a concordância é a discussão das divergências entre os observadores e não só incluir novas incidências radiológicas como rotina na avaliação das fraturas distais do rádio. Além disso, consideraram importante que antes de passar a utilizar a classificação completa é necessário resolver as divergências que ocorrem entre as três classes $A, B$ e $C$.

Comparando-se nossos resultados da concordância intra e interobservador tanto da classificação de Frykman como da classificação $A O$, observamos que foram semeIhantes aos encontrados por outros autores (Tabelas 5 e 6). Com relação à classificação Universal não pudemos fazer comparação, uma vez que não havia, até o momento, outros estudos sobre sua reprodutibilidade.

Diante dos resultados apresentados neste estudo, obter um padrão ouro para estas classificações torna-se uma tarefa difícil; dessa maneira, qualquer comparação de diferentes séries por diferentes autores torna-se duvidosa.

A classificação de qualquer fratura seria ideal se fornecesse informação suficiente para se escolher um tratamento adequado, determinar o prognóstico, comparar diferentes modalidades de tratamentos, ter uma reprodutibilidade satisfatória, ser de fácil memorização e importante para a educação do ortopedista em formação, ser aceita internacionalmente e levar em consideração o estado geral do paciente e das partes moles que envolvem a fratura ${ }^{6,8,13}$. 
Do ponto de vista da reprodutibilidade, constatamos que as classificações avaliadas não foram satisfatórias, mas devemos continuar estudando-as para que tenhamos, cada vez mais, um melhor entendimento das fraturas distais do rádio.

\section{Conclusóes}

Para as três classificações avaliadas houve melhora da concordância intra-observador associada com o maior grau de experiência dos observadores, mas na concordância interobservador a experiência não influenciou na meIhora dos resultados.

A classificação de Frykman não deve ser utilizada por apresentar muitas limitações e baixos índices de reprodutibilidade interobservador.

Devido a sua complexidade e baixos índices de reprodutibilidade, tanto intra como interobservador, a classificação $\mathrm{AO}$ tem seu uso comprometido.

Apesar de considerar aspectos importantes das fraturas distais do rádio e da sua simplicidade, a classificação Universal tem também seu uso comprometido devido aos baixos valores encontrados na sua reprodutibilidade interobservador.

\section{SUMMARY}

Distal Radius fRActures: CONSISTENCY OF THE CLASSIFICATIONS

OBJECTVE. The purpose of this study was to determine the intra-observer and interobserver reproducibility of Frykman, AO and Universal classifications for the fractures of the distal radius.

Methods. In this study, 40 radiographs of fractures of the distal radius were selected and classified by orthopedists of different centers and levels of experience, determining the intraobserver and interobserver reproducibility of the classifications using Kappa statistic method.

REsults. The medium intra-observer concordance observed was moderate to Frykman and Universal classifications and light to the $A O$ system. The medium interobserver reproducibility was light in the two readings to Frykman and Universal classifications and despicable in the second reading of the $A O$ classification.

Conclusion. All the classifications used presented questionable interobserver reproducibility compromising the use of the three evaluated systems. [Rev Assoc Med Bras 2004; 50(l): 55-6I]

KeY Words: Classifications. Distal radius. Fractures. Reproducibility.

\section{REFERÊNCIAS}

I. Mallmin H, Ljunghall S. Incidente of Colles'fracture in Uppsala. A prospective study of a quarter-million population. Acta Orthop. Scand 1992; 63:213-5.

2. Graff $\mathrm{S}$, Jupiter J. Fracture of the distal radius: classification of treatment and indications for external fixation. Injury 1994; 25(suppl 4): 1 4-25.

3. Porter MI, Stockley, I. Fractures of the distal radius. intermediate and end results in relation to radiologic parameters. Clin Orthop 1987; 220:24I-52.

4. Cooney WP, Dobyns JH, Linscheid RL. External pin fixation for instable Colles' fractures. J. Bone Joint Surg 1979; 6|A:840-5.

5. Fernandez DL, Geissler WB, Lamey DM. Wrist instability with or following fractures of the distal radius. In: Büchler U. Wrist Instability. Bern: Martin-Dunitz; 1996. p. I8I-92.

6. Müller ME. The principle of the classification. In: Müller ME, Allgöwer M, Schneider R, Willenegger $\mathrm{H}$. Manual of internal fixation: techniques recomendad by the AO-ASIF group. New York: Springer-Verlag; 1991. p. 118.

7. Frykman G. Fracture of the distal radius, including sequilla of shoulder-hand síndrome: disturbance of the distal radio-ulnar joint and impairment of nerve function. A clinical and experimental study. Acta Orthop Scand Suppl 1973; 108: I.

8. Burstein $\mathrm{AH}$. Fracture classification systems: do they work and are they useful? (editorial). J Bone Joint Surg 1993; 75A: 1743-4.
9. Kreder HJ, Hanel DP, Mckee M, Jupiter J, Mcgillivary G, Swiontkowski MF. Consistency of $\mathrm{AO}$ fracture classification for the distal radius. J. Bone Joint Surg [Br] 1996; 78B:726-31.

I0. Jupiter JP, Fernandez DL. Comparative classification for fractures of the distal end of the radius. J Hand Surg [Am] 1997; 22:563-7|

II. Oskam J, Kingma J, Klasen HJ. Interrater reliability for the basic categories of the $\mathrm{AO} /$ ASIF system as a frame of referente for classifying distal radial fractures. Percept Mot Skills 200 I; 92:589-94.

12. Cooney WP. Fractures of the distal radius: a modern treatment- based classification. Orthop Clin North Am 1993; 24:21 I-6.

13. Berstein J. Taxonomy and treatment - a classification of fracture classifications. J. Bone Joint Surg [Br] 1997; 79B:706-7.

14. Tenório RB, Mattos CA, Araújo LHC, Belangero WD. Análise da reprodutibilidade das classificações de Lauge-Hansen e DanisWeber para fraturas de tornozelo. Rev Bras Ortop 2001; 36:434-7.

15. Landis JR, Koch GK. The measurement of observer agreement for categorical data. Biometrics 1977; 33:159-74.

16. Statxact for Windows Statistical Software for exact nonparametric inference. Cytel Software Corporation. Cambridge; 1977.

17. Gusmão PDF, Mothes FC, Rubin LA, Gonçalves RZ, Telöken MA, Schwartsmann CR. Avaliação da reprodutibilidade da classificação de Garden para fraturas do colo femoral. Rev Bras Ortop 2002; 37:387-91.

18. Andersen DJ, Blair WF, Steyers CM Jr, Adams BD, El-Khouri GY, Brandser EA. Classification of distal radius fractures: an analysis of interobserver reliability. I Hand Surg [Am] 1996:2 I (4):574-82.

19. Illarramends A, González Della ValleA, Segal E, De Carli P, Maignon G, Gallucci G. Evaluation of simplified Frykman and $A O$ classifications of fractures of the distal radius. Int Orthop 1998; 22:1 II-5.

20. Flinkkilä T, Nikkola-Sihto $A$, Kaarela $\bigcirc$, Pääkkö $E$, Raatikaine T. Poor interobserver reliability of ao classification of fractures of the distal radius. additional computed tomography is of minor value. J Bone Joint Surg [Br] 1998; 80B:670-2.

21. Cooney WP. Fractures of the distal radius: a modern treatment- based classification. Orthop Clin North Am 1993; 24:21 I-6.

Artigo recebido: 18/12/02 Aceito para publicação: 17/09/03 